\title{
Réseaux sociaux, entre médias et médiations, des espaces à méditer plutôt qu'à médire
}

Olivier Le Deuff

\section{(2) OpenEdition \\ 12 Journals}

Édition électronique

URL : http://journals.openedition.org/communicationorganisation/4091

DOI : 10.4000/communicationorganisation.4091

ISSN : 1775-3546

Éditeur

Presses universitaires de Bordeaux

\section{Édition imprimée}

Date de publication : 1 juin 2013

Pagination : 5-12

ISBN : 978-2-86781-883-7

ISSN : $1168-5549$

\section{Référence électronique}

Olivier Le Deuff, « Réseaux sociaux, entre médias et médiations, des espaces à méditer plutôt qu'à médire », Communication et organisation [En ligne], 43 | 2013, mis en ligne le 01 juin 2013, consulté le 22 septembre 2020. URL : http://journals.openedition.org/communicationorganisation/4091 ; DOI : https://doi.org/10.4000/communicationorganisation.4091 


\title{
Réseaux sociaux, entre médias et médiations, des espaces à méditer plutôt qu’à médire
}

\author{
Serge Agostinelli', Olivier Le Deuff ${ }^{2}$
}

Fréquemment mis en avant pour des réussites de leurs dirigeants ou au contraire pour leurs dérives, les réseaux sociaux sont parvenus à gagner peu à peu en légitimité. Celle-ci favorise une pénétration de plus en plus avérée dans toutes les couches de la population et, en tant que dispositifs ancrés dans le réel, ils amplifient les évènements quotidiens et l'actualité. Toutefois, les réseaux sociaux recouvrent différentes réalités et leurs frontières semblent peu étanches. Les fonctionnalités sociales accompagnent désormais un grand nombre d'applications et de services, tandis que les possibilités d'associer des actions et réactions (commentaires, likes) avec les comptes de réseaux sociaux les plus emblématiques sont devenues courantes. Leur diffusion dans différentes sphères d'activités autant personnelles que professionnelles mérite un examen particulier que ce numéro tente d'effectuer en étudiant les réseaux sociaux numériques mais aussi les médiations sociales associées aux pratiques. Réseaux sociaux numériques voire réseaux socionumériques (Rsn), médias sociaux, les expressions se sont multipliées avec diverses définitions et des réalités différentes entre les dispositifs mais aussi les usages parfois selon les pays. Difficile aussi de dire qu'un réseau ou un média n'est pas social, ce que rappelaient à juste titre Alexandre Coutant et Thomas Stenger (2011) qui en appelaient à une prise en compte de la complexité qui étudie autant les aspects techniques que les interactions sociales et humaines à l'œuvre. Malgré des dimensions réellement innovantes et des effets massifs dans la mise en relation d'un grand nombre de personnes, il convient de rappeler que les communautés en ligne sont bien antérieures (Rheingold, 2013). Cet esprit communautaire constitue probablement déjà un mode de fonctionnement qui a accompagné le développement du réseau internet, ce que montre Fred Turner dans sa biographie de Steward Brand (Turner, 2012). La question des réseaux sociaux a été beaucoup abordée sous l'angle de l'étude des sociabilités

1 LSIS, UMR CNRS 7296, Professeur à l'Université Aix-Marseille ; serge.agostinelli@univ-amu.fr

2 Maître de conférences en sciences de l'information et de la communication. Laboratoire MICA, EA 4426, Université de Bordeaux 3 ; oledeuff@gmail.com 
notamment juvéniles avec les travaux de danah boyd (Boyd, 2006), ainsi que par des travaux autour de l'identité numérique et des phénomènes de surveillance (Lyon, 2007) qui résultent de la publication par les internautes de profils qui constituent des bases de données d'intentions. Beaucoup d'études ont concerné les principaux réseaux comme Facebook ou plus récemment Twitter et son impact politique. Cette inclusion des pratiques sociales de réseaux numériques dans la vie quotidienne et l'intérêt qu'ils suscitent dans plusieurs champs disciplinaires, entrainent une nécessaire modestie quant à la mesure réelle de la portée de ces dispositifs. La dissémination des technologies sociales opère des transformations dans la société et dans les existences qui font de ces réseaux des objets techniques doubles, autant remèdes que poisons (Stiegler, 2011). Ce numéro ne prétend donc aucunement à l'exhaustivité et cherche davantage à mettre en avant des saillances et des éléments à saisir et à développer notamment pour les sciences de l'information et de la communication. Beaucoup de concepts et de données issus des recherches sur les réseaux sociaux s'avèrent pertinents pour les SIC. On peut citer pêle-mêle, l'identité numérique et les enjeux de formation associée, l'e-réputation, les médiations informationnelles et communicationnelles qui s'y opèrent, les conséquences sur les organisations, les nouvelles formes de communication qui en résultent, les transformations sur le monde professionnel ou bien encore les impacts sur la communication scientifique et l'information scientifique et technique. De nombreux domaines et concepts qui intéressent les SIC sont inévitablement impactés. Inévitablement, impossible de faire l'impasse sur l'influence technique, sur les logiques d'usages, sur les affordances ainsi que sur les cultures et pratiques des différents usagers.

\section{Des réseaux sociotechniques}

Difficile par conséquent de dissocier les interactions sociales qui s'opèrent des dispositifs techniques mobilisés. Le côté numérique rappelle un caractère technique important dans sa matérialité mais aussi dans les contraintes et opportunités apportées par les dispositifs logiciels qui en font des objets qui ne peuvent en aucun cas être qualifiés de neutres sans pour autant prétendre que les affordances empêchent tout caractère évolutif car le succès parvient souvent des possibilités d'innovation ascendante voire d'imprévus. Le cas du hashtag, ce mot-clé précédé d'un dièse, issu des pratiques folksonomiques, est un bon exemple d'un succès que les créateurs de Twitter n'avaient pas envisagé lors de la conception du réseau.

Le numéro interroge à cet effet le réseau sur son caractère innovant et plus particulièrement sur ses potentialités de médiation voire de formation de l'individu ou tout au moins de son e-réputation. Mais cette médiation peut être également de nature documentaire notamment pour les organisations qui cherchent ainsi à gérer les informations et les documents qui les concernent. Un projet particulièrement important pour les municipalités qui cherchent 
à mieux informer et communiquer de façon différente en profitant de l'opportunité parfois de toucher de nouveaux publics.

Les réseaux sociaux apparaissent alors comme des objets techniques dont la complexité sociotechnique oblige à penser l'individu, mais aussi les collectifs dans une relation avec les dispositifs qu'on pourrait qualifier de transductive dans la lignée de Simondon (1989) dans la mesure où s'opèrent des co-constructions. Il convient dès lors de rappeler que l'individu social ne peut exister sans l'individu technique et réciproquement. D'où l'intérêt pour une ontologie variable des actants capable de mieux distinguer les possibilités d'actions des actants humains, notamment leur capacité à modifier et à faire évoluer les dispositifs socio-techniques.

\section{Des intérêts pour la recherche}

L'intérêt scientifique est évident à condition de ne pas demeurer uniquement sur une vision socialisante. En effet, les dispositifs permettent d'envisager de nouveaux types d'éditorialisation notamment collaborative des évènements comme les colloques, tant il s'agit de plus en plus de pouvoir commenter, annoter et écrire collectivement. Les dispositifs sociaux sont donc autant des objets d'études que des moyens de communication scientifique.

L'étude de plus en plus fréquente des réseaux sociaux et des données issues de ces réseaux mérite un regard critique, particulièrement en ce qui concerne le développement des visualisations. Riches en potentialités, les études des graphes qui renouent avec les théories premières des réseaux sociaux comportent toutefois des limites et des lacunes qui sont ici exposées et discutées afin de pouvoir envisager des sciences sociales computationnelles de qualité, des humanités socionumériques qui permettent autant le faire que la réflexion.

Par conséquent, les réseaux sociaux constituent désormais des espaces d'études mais aussi de réalisations à part entière pour la recherche. Si la tendance a parfois été de les médire voire de les considérer comme des modes passagères, l'intégration croissante dans les pratiques y compris des chercheurs en font des lieux incontournables pour réaliser des études, des mesures à de nouvelles échelles. L'enjeu est alors de savoir se positionner à la bonne distance pour mieux observer les phénomènes ("not tool but lens ", comme le recommande Alan Liu (Liu, 2009, p. 22), une recommandation primordiale pour le développement des digital studies ou des digital humanities. Ce numéro tente d'apporter des pistes de réflexion et de méthodes pour continuer à entreprendre des études au sein de ces entrelacs numériques porteurs de marques, de traces, conscientes et inconscientes, car "méditer, sans traces, devient évanescent $»^{3}$.

3 Evidemment, cette phrase inspirée de Mallarmé est quelque peu détournée de l'orignal : «Ton acte toujours s'applique à du papier ; car méditer, sans traces, devient évanescent, ni que s'exalte l'instinct en quelque geste véhément et perdu que tu cherchas. " (Stéphane Mallarmé, "Quant au livre » in Divagations. Mais il est 


\section{À méditer.}

\section{Présentation des articles}

Le premier article d'Alain Barrat, "La notion de réseau complexe : du réseau comme abstraction et outil à la masse de données des réseaux sociaux en ligne » montre l'intérêt des études sur les réseaux sociaux pour examiner des corpus de données à grande échelle. Il replace l'étude du réseau depuis ses formes les plus anciennes en rappelant que le réseau ou graphe peut être simplement défini comme un ensemble "de points, appelés sites ou sommets, reliés par des liens ». Il repositionne donc les études sur les réseaux sociaux dans cette lignée scientifique de l'étude du réseau. Il montre que les potentialités de recueil de données s'accroissent par des mécanismes de captation sophistiqués qui appellent des études complexes sur des données parfois massives. Dans tous les cas, cela implique "clairement la nécessité d'approches multi-disciplinaires associant sciences humaines et sociales aux sciences dures " pour une exploitation des données en évitant des biais possibles et en ouvrant des perspectives de recherche augmentée et probablement trandisciplinaire.

Mylène Hardy, Serge Agostinelli en appellent à un renouvèlement épistémologique pour mieux appréhender l'hétérogénéité. Dans leur article, "L'ontologie variable des actants : pour une épistémologie renouvelée dans les recherches sur les réseaux », ils préconisent notamment une meilleure prise en compte des actants sociaux et humains dans leur capacité de réaction, de modification et de transformation des dispositifs.

Pierre-Michel Riccio dans son article "Vers un modèle d'efficience des collectifs » propose justement une démarche appliquée aux domaines de la transmission et des acquisitions de compétences qui aille bien au-delà du seul CMS idéal pour mieux cerner les possibilités d'échanges entre scientifiques afin de produire un modèle d'intelligence collective.

Le cas des pratiques transmédias est étudié par Mélanie Millette dans son article "Pratiques transplateformes et convergence dans les usages des médias sociaux ». Elle cherche à comprendre les pratiques transplateformes de partage et de diffusion effectuées par les usagers sur les réseaux sociaux. C'est l'exemple des podcasters qui est ici retenu. Ils possèdent souvent des compétences plus expertes que l'usager moyen et se montrent plus à même de mieux comprendre les stratégies transplateformes qu'ils utilisent notamment via les réseaux sociaux comme Facebook. Le chercheur doit toutefois développer des stratégies complexes pour suivre la traçabilité de diffusion vers plusieurs médias sociaux ce qui se révèle parfois complexe.

très tentant de considérer que le numérique se substitue ici au support papier et que quelque part désormais, même un acte irréfléchi peut « s'accrocher » au numérique et produire une trace. On songe notamment ici à l'impulsion du like. Voir Olivier Le Deuff. Du tag au like. La pratique des folksonomies pour améliorer ses méthodes d'organisation de l'information, Fyp éditions, 2012. 
L'article de Jean-Christophe Plantin "Qu'y a-t-il à côté d'un graphe de sites web? Analyse de réseaux sociaux et pratiques de synchorisation en ligne» développe une réflexion critique et méthodologique sur le recours à l'analyse des réseaux sociaux pour la recherche en SIC en examinant la méthode des graphes. I1 mobilise à cet effet deux concepts, la synchorisation en tant que "processus qui consiste à se donner un espace commun pour être et pour agir " (Beaude, 2012) et l'écume adapté aux SIC (Rieder, 2010) afin que les résultats obtenus par les méthodes des graphes ne deviennent pas une fin en soi mais, au contraire, la possibilité de prolonger et d'accompagner les résultats par d'autres moyens.

Impossible de ne pas évoquer dans ce numéro, les enjeux très actuels de la fameuse e-réputation, ce que réalise Camille Alloing dans son article « La médiation documentaire sur les réseaux socionumériques comme vecteur de l'e-réputation? La Poste, Twitter et les agents-facilitateurs ». Camille Alloing considère les individus comme des agents de pertinence capables de devenir des intermédiaires pour opérer une médiation documentaire entre information et communication. L'originalité est davantage de s'attarder à l'e-réputation des organisations qui procède de formes de redocumentarisations itératives et qui s'appuie sur les réseaux sociaux. Les organisations décrites ici tentent de réaliser une "médiation documentaire : pour lier l'information produite à son encontre à la communication qu'elle soubaite développer».

C'est aussi le cas des municipalités qui se penchent sur leurs dispositifs de communication, ce qu'analyse Ewa Krzatala-Jaworska dans son article «Les municipalités à la conquête des réseaux sociaux, mais pourquoi faire ? ». Partant du constat d'une présence accrue et d'un intérêt évident pour les réseaux sociaux dans les stratégies de communication des municipalités, elle cherche les raisons d'un tel engouement et tente de mettre en avant les stratégies mises en place. Les degrés de participation des citoyens sont parfois faibles voire inexistants mais les municipalités semblent miser également sur des stratégies de mises en visibilité et de compétitivité par rapport aux municipalités voisines afin de témoigner d'un véritable investissement en matière numérique. Les résultats peuvent être alors parfois décevants faute d'un investissement humain suffisamment pensé notamment en matière de médiation et de stratégie voire simplement budgété avec un recrutement approprié.

Louise Merzeau étudie un dispositif innovant et expérimental mis en place par l'IRI dans le cadre des entretiens des nouveaux mondes dans son article "Éditorialisation collaborative diun événement. L'exemple des Entretiens du nouveau monde industriel 2012 ». Elle montre que les réseaux sociaux doivent être aussi considérés comme des dispositifs d'éditorialisation collective. Pour cela, elle étudie le dispositif mis en place qui rassemble plusieurs outils aux fonctionnalités sociales comme storify, pearltrees et notamment PolemicTweet développé par l'IRI qui s'appuie sur Twitter et qui vise à catégoriser les tweets "selon 4 modalités: question, référence, accord ou désaccord». Louise Merzeau met 
en avant les enjeux autour de ces dispositifs transmédiatiques qui loin d'être les instruments d'une désintermédiarisation et d'une disparition des documents, se révèlent surtout être des espaces de l'exercice d'une translittératie.

Alexandre Coutant interroge les potentialités innovantes des réseaux sociaux dans son article "Quelle place pour l'innovation dans les médias sociaux? ". Il rappelle qu'il s'agit d'un secteur innovant particulier d'autant que face aux discours positivistes fréquemment relayés dans les médias à propos des gains des principaux réseaux, il convient de mettre en face le cimetière de projets qui ne cesse de croître. Alexandre Coutant montre que le côté participatif indispensable à tout projet d'innovation, notamment ascendante, est souvent réducteur car les projets finissent par négliger "la diversité des zones que recouvrent les médias sociaux et par conséquent les possibilités de se différencier ». La cartographie proposée dans l'article permet de mieux appréhender ces possibilités.

Une interview du directeur général de Viadéo, Olivier Fecherolle par Thomas Stenger et Alexandre Coutant vient d'ailleurs interroger la question de l'intégration des réseaux sociaux dans les pratiques professionnelles. Initialement conçu comme point d'appui pour les ressources humaines, le réseau social présente des opportunités en matière de marketing de par des possibilités de filtrage et de ciblage extrêmement précises.

Ces pratiques professionnelles sont également analysées par Francine Charest, Anne-Marie Gauthier, François Grenon qui décrivent « une démarche pour réussir l'appropriation et l'intégration des médias sociaux dans une stratégie de communication corporative » dans leur article «Appropriation et stratégies d'intégration des médias sociaux par les professionnels de la communication ».

\section{BIBLIOGRAPHIE}

BEAUDE B., Internet, changer l'espace, changer la société, FYP éditions, 2012.

BOYD D., «Why Youth (Heart) Social Network Sites: The Role of Networked Publics in Teenage Social Life », in D. BUCKINGHAM Ed., Youth, Identity, and Digital Media, Cambridge, MIT Press, 2008, p. 19-42.

CLARKE R., "The digital persona and its application to data surveillance», Information Society, 10:2, 1994, p. 77.

LIU A., " Digital Humanities and Academic Change », English Language Notes, 47, Spring-Summer 2009, p. 17-35.

LYON D., Surveillance Studies: An Overview, Polity, 2007.

RHEINGOLD H., Les communautés virtuelles, Addison-Wesley France, 1996.

RIEDER B. « De la communauté à l'écume : quels concepts de sociabilité pour le "web social" ? ", TIC E Société, 2010, Vol. 4, n 1, Revue en ligne : http://ticetsociete. revues.org/822.

SIMONDON G., Du mode d'existence des objets techniques, Paris, Aubier, 1989. 
STENGER T., COUTANT A., "Introduction », Hermès, n59 " Ces réseaux numériques dits sociaux ", p. 9-17, 2011.

STIEGLER B. et al., Réseaux sociaux : Culture politique et ingénierie des réseaux sociaux, FYP éditions, 2012.

TURNER F., Aux sources de l'utopie numérique: De la contre culture à la cyberculture., C\&F Editions, 2012.

\section{Comité scientifique du numéro}

Dominique Augey : PU, Aix-Marseille-Université

Virginie de Barnier, PU, IAE, Aix-Marseille Université

Philippe Bouquillon, PU, Université Paris 13

Mélanie Bourdaa, Mcf en SIC, Université de Bordeaux 3

Yves Chevalier, Pr. Emerite en Sic, Université de Bretagne Sud

Eric George, PU, Université Québec, Montréal

Jean-Claude Hennet, DR, Aix-Marseille Université

Bernard Jacquemin, Mcf en Sic, Université de Lille 3

Valérie Jeanne-Perrier, MCF, Celsa, Paris Sorbonne

Feirouz Lima, Mcf en SIC, Université de Franche Comté

Vincent Liquète, Pr. en SIC, Université de Bordeaux 4

Nathalie Pinède, Mcf en SIC, Université de Bordeaux 3

Bernhard Rieder, professeur associé à l'Université d'Amsterdam

Denis Ruellan, PU, Université Rennes 1

Nathalie Sonnac, PU, IFP, Univ. Paris 2 Panthéon-Assas

Pierre Trudel, Chaire L.R. Wilson - Université Montréal

Nayra Vacaflor, docteur en Sic, IGr, Université de Bordeaux 3 
